\title{
Advanced Ultrasonic Structural Monitoring of Waveguides
}

\author{
M. Cammarata ${ }^{1 a}$, D. Dutta ${ }^{2, b}$, H Sohn $^{3, c}$, P. Rizzo ${ }^{* 1, d}$ and K. A. Harries ${ }^{1, e}$ \\ ${ }^{1}$ Dept. of Civil and Environmental Engineering, University of Pittsburgh, 949 Benedum Hall, \\ Pittsburgh, PA 15261.
}
${ }^{2}$ Dept. of Civil and Environmental Engineering, Carnegie Mellon University, 5000 Forbes Avenue, Pittsburgh, PA, USA

${ }^{3}$ Dept. of Civil and Environmental Engr., Korea Advanced Institute of Science and Technology, Daejeon, 305-701, Korea

amstaxmarcello@yahoo.it, ${ }^{\mathrm{b}}$ ddutta@cmu.edu , ${ }^{\mathrm{C} h s o h n @ c m u . e d u},{ }^{d}$ pir3@pitt.edu, ekharries@pitt.edu

Keywords: Ultrasonic guided waves, nonlinear acoustics, feature extraction, Discrete Wavelet Transform.

\begin{abstract}
Ultrasonic Guided Waves (UGWs) are a useful tool in those structural health monitoring applications that can benefit from built-in transduction, moderately large inspection ranges and high sensitivity to small flaws. This paper describes two methods, based on linear and nonlinear acoustics for structural damage detection based on UGWs. The linear method combine the advantages of UGW inspection with the outcomes of the Discrete Wavelet Transform (DWT) that is used for extracting defect-sensitive features that can be combined to perform a multivariate diagnosis of damage. In particular, the DWT is exploited to generate a set of relevant wavelet coefficients to construct a uni-dimensional or multi-dimensional damage index that, in turn is fed to an outlier algorithm to detect anomalous structural states. The nonlinear acoustics method exploits the circumstance that a cracked medium exhibits high acoustic nonlinearity which is manifested as harmonics in the power spectrum of the received signal. Experimental results also indicate that the harmonic components increase non-linearly in magnitude with increasing amplitude of the input signal. The proposed nonlinear technique identifies the presence of cracks by looking at the harmonics and their nonlinear relationship to the input amplitude. The general framework presented in this paper is applied to the detection of fatigue cracks in an I-shaped steel beam. The probing hardware consists of Lead Zirconate Titanate (PZT) materials used for both ultrasound generation and detection at chosen frequency. The effectiveness of the proposed methods for the structural diagnosis of defects that are small compared to the waveguide cross-sectional area is discussed.
\end{abstract}

\section{Introduction}

Steel structures are ubiquitous in mechanical, industrial and civil engineering systems. Failure of these structures is often attributed to fatigue or fracture cracks that can develop for instance at the flange-web junction of a bridge girder, in a railway track, or in the sub-structures of a power generation plant. In most cases cracks cannot be avoided, thus there is a need for non-destructive inspection (NDI) or structural health monitoring (SHM) technique aimed at detecting structural deficiencies at early stage of deterioration.

NDI techniques such as acoustic emission [1], eddy current [2], ambient-vibration based [3], and impedance-based methods [4] were proposed for the detection of cracks in steel structures. Recently methods based on ultrasonic guided waves (UGWs) gained popularity owing to the capability of inspecting moderately large areas at once from a single probe attached or embedded in the structure and of possessing high sensitivity to small flaws [5-7]. UGWs and particularly Lamb waves were successfully used to monitor the propagation of fatigue cracks in metallic structures [8-10].

\footnotetext{
Corresponding author
} 
In the last few years the necessity for improvements in the field of signal conditioning, feature extraction, and defect classification became evident in order to enhance the detection, sizing, location, and classification of the monitoring scheme.

This paper describes two methods based on linear and nonlinear acoustics for structural damage detection based on ultrasonic guided waves (UGWs). The linear method combines the advantages of UGW inspection with the outcomes of the Discrete Wavelet Transform (DWT). In particular, the DWT is exploited to generate a set of relevant wavelet coefficients to construct a uni-dimensional or multi-dimensional damage index that is fed to an outlier algorithm [11] to detect anomalous structural states. The nonlinear acoustics method instead correlates defects with the presence of additional frequency components in the output signal. The approach exploits the circumstance that a cracked medium exhibits high acoustic nonlinearity which is manifested as harmonics in the power spectrum of the received signal [12-13]. The generation of super-harmonics due to cracks is often attributed to the phenomenon of crack opening and closing caused by traveling acoustic waves [14]. The general framework presented in this paper is applied to the detection of fatigue cracks in a W6x15 steel beam. The probing hardware consists of wafer piezoelectric transducers (PZTs) made of Lead Zirconate Titanate materials used for both ultrasound generation and detection at chosen frequency. These PZTs are suitable in SHM application as they are intrinsically easy to attach to the structure surface or to embed in the structure.

\section{Experimental setup}

The experiment was performed on a $2.74 \mathrm{~m}$ long W6 x 15 (SI: W150 x 22.5) steel beam. The dimensions of the steel specimen are shown in Figure 1. Two notches were cut into the bottom (tension) flange near the center of the beam-span as shown in Figure 1b. These notches served as fatigue crack initiators, and also helped to increase the stress at this section in order to accelerate the development of fatigue cracks. The notches were designed to have a theoretical fatigue life on the order of 40,000 cycles at an applied stress range of $190 \mathrm{MPa}$.

Three PSI-5A4E type PZT wafer transducers $(1.0 \mathrm{~cm} \times 1.0 \mathrm{~cm} \times 0.0508 \mathrm{~cm})$ were mounted on the bottom flange of the beam. One transducer acted as a transmitter and was placed in between the two sensors which were located $500 \mathrm{~mm}$ apart (Figure 2). The crack initiator fell between the transmitter and sensor $S_{1}$. The suite of instrumentation was completed by four electrical resistance crack gages placed to monitor the onset and growth of the fatigue cracks. These gages were placed at the notch root on both sides of the flange (Figure 2).

To produce the cracks, the beam was loaded in simple mid-span loading over a span length of 2.74 $\mathrm{m}$. The midspan load was cycled from $4.5 \mathrm{kN}$ to $40.5 \mathrm{kN}$ resulting in a load range of $36 \mathrm{kN}$. Cycling was carried out at a rate of $1 \mathrm{~Hz}$. The $36 \mathrm{kN}$ applied load corresponds to a tensile stress range of 190 $\mathrm{MPa}$ at the notch root of the tension flange. Figure 3 shows the history of crack propagation in both west and east flanges of the steel beam.

(a)

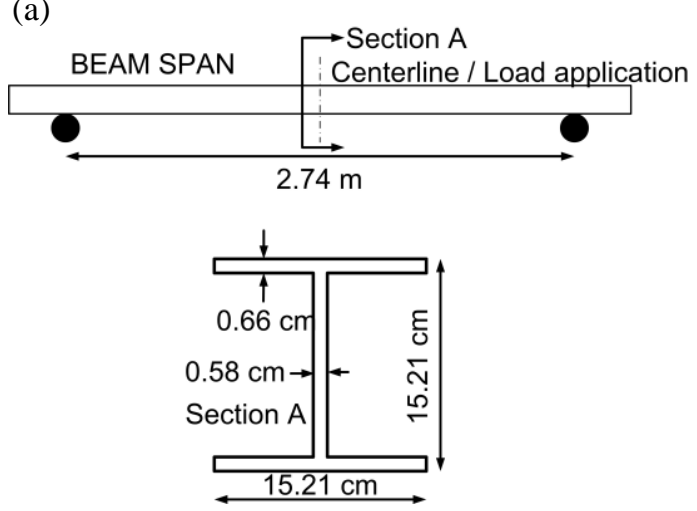

ELEVATION
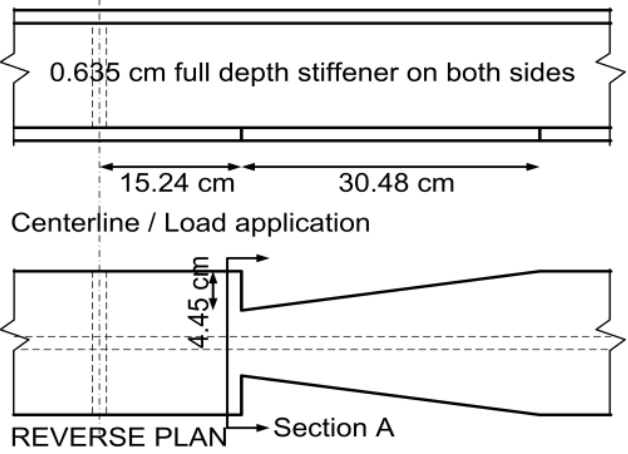

(b)

Figure 1 - Dimensions of the steel W6x15 (SI: W150 x 22.5) section: (a) Beam span and cross section. (b) Elevation and plan views of the flange in the tension side. 


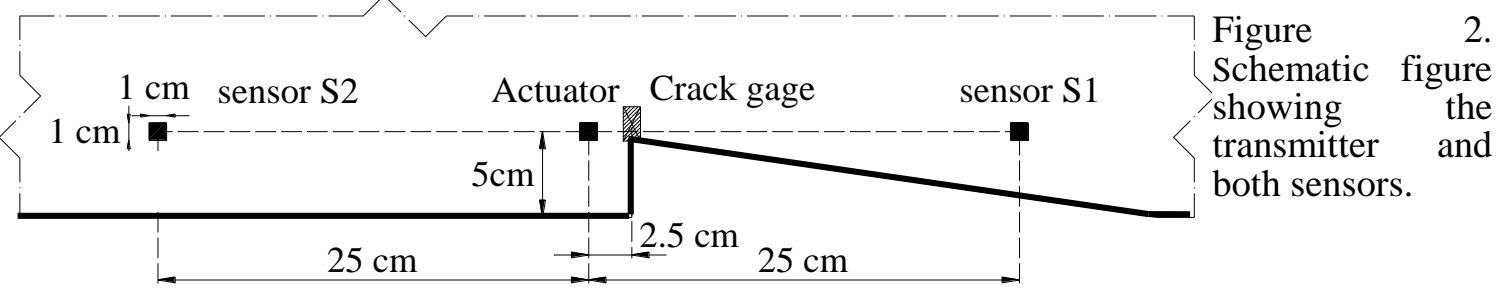

\section{Linear approach}

A National Instruments PXI ${ }^{\circledR}$ unit running under LabVIEW ${ }^{\circledR}$ was employed for signal excitation, detection and acquisition. Five-cycle 10 Volts peak-to-peak (Ppk) $225 \mathrm{kHz}$ toneburst modulated with a Hanning window was excited. The first symmetric $S_{0}$ and the first anti-symmetric $A_{0}$ mode were excited. The acquired waveforms were corrupted 19 times with white Gaussian noise in order to increase the statistical population. Thus 260 time histories were available. Each time waveform was windowed in order to separate the $S_{0}$ from the $A_{0}$ mode. Ultrasonic signals were processed through the DWT, which decomposes the original time-domain signal by computing its correlation with a short-duration wave called the mother wavelet that is flexible in time and in frequency. The decomposition phase transforms the function into wavelet coefficients following hierarchical steps (levels) of different time and frequency resolution. Each level contains the signal information both in the time and the frequency domain over a certain frequency bandwidth. De-noising of the original signal can be achieved if only a few wavelet coefficients, representative of the signal, of one or more levels are retained and the remaining coefficients, related to noise, are discarded. The Daubechies mother wavelet of order 40 ( $\mathrm{db} 40)$ was considered and the eight largest wavelet coefficient moduli from the decomposition level associated were retained. Several UGW-based statistical features were extracted from the unprocessed waveform (time domain) and the wavelet coefficient vector (joint time-frequency domain). The index is defined as the ratio between a certain feature $F_{\mathrm{S} 2}$ calculated from the signal detected by sensor $S_{2}$, over the same feature $F_{\mathrm{S} 1}$ extracted from sensor $S_{1}$. As the notch is located in the section of the beam between the actuator and the sensor S1 the value of D.I. is expected to increase compared to its normal, defect-free value (nominally one).

The damage indices were then considered for outlier analysis. An outlier is a datum that appears inconsistent with a set of data, the baseline that describes the normal condition of the structure under investigation. In the analysis of one-dimensional elements, the detection of outliers is based on the determination of the discordancy between the one-dimensional datum and the baseline. One of the most common discordancy tests is based on the deviation statistics, $z_{\xi}$, defined as:

$$
z_{\zeta}=\frac{\left|x_{\zeta}-\bar{x}\right|}{\sigma}
$$

where $x_{\zeta}$ is the potential outlier, and $\bar{x}$ and $\sigma$ are the mean and the standard deviation of the

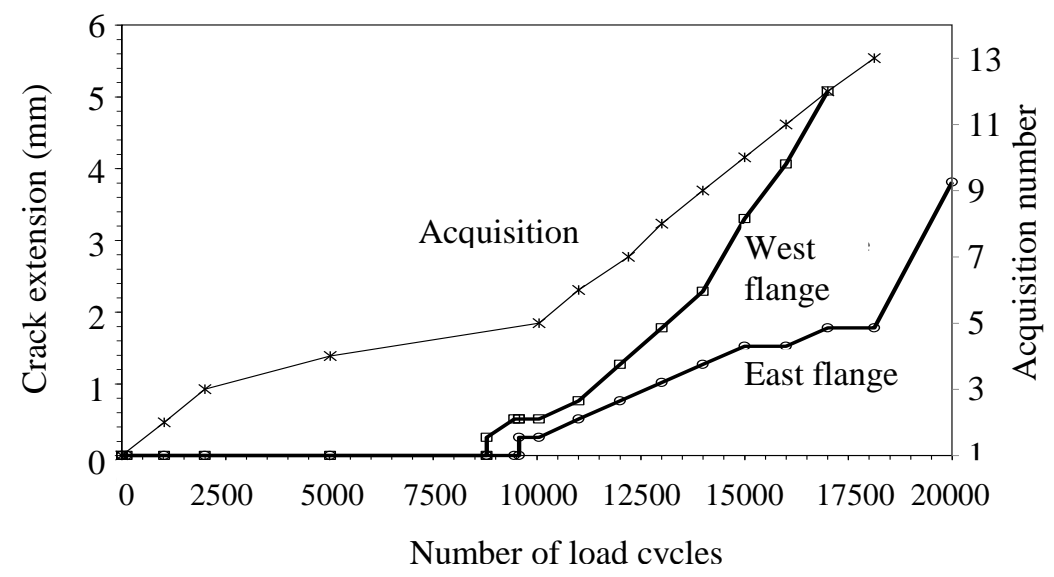

Figure 3 Propagation history of the crack emanating from the notch-tip of west and east part of the tension flange of the steel beam. The acquisition number history is superimposed. 
baseline, respectively. The value of $z_{\zeta}$ is then compared to a threshold value to determine whether the datum is an outlier (falling above the threshold) or not. The baseline was computed from the first 60 (=3 acquisitions $\mathrm{x}(1$ original +19 corrupted) $)$ time histories that, according to Fig. 3, were representative of the pristine structure at load cycles 0,1250 , and 2500 , respectively. Once the values of $\bar{x}$ and $\sigma$ of the baseline distribution were determined, the threshold value was taken as the upper value of $3 \sigma$, equal to $99.73 \%$ of the Gaussian confidence limit.

Figure 4 shows the discordancy values as a function of the sample number. The damage index computed from three statistical features extracted from the wavelet coefficient vector were considered. These features are the root mean square (RMS), the Ppk, and the K factor. Figs. 8a and $8 \mathrm{~b}$ refer to the propagation of the $\mathrm{A}_{0}$ and $\mathrm{S}_{0}$, respectively. Clear steps are visible almost at every 20 sample numbers corresponding to the progressive increase of the crack. In the samples range 61-80 twenty outliers were detected. These data are associated to the acquisition at 5,000 cycles. According to Fig. 3 the beam was still in its pristine conditions. Thus, these outliers are classified as false positives. The remaining 180 samples properly indicate damaged conditions of the structure. By observing Fig. 8 the damage index from each feature possesses a different sensitivity to the presence of damage. Qualitatively the discordancy increases with increasing defect size, however, it can be seen that the sensitivity to damage (represented by the rate of change of the discordancy plot) is larger for the K-factor-based damage index applied to the $\mathrm{S}_{0}$ mode (Fig. 8b) and the RMS-based damage index applied to the $\mathrm{A}_{0}$ mode (Fig. 8d). Sometime the damage index cannot be used to discriminate between two adjacent acquisitions. This is due to the fact that the difference in size between such defects is so small and lies in a few wavelet coefficients of lower amplitude that do not affect the extreme values.
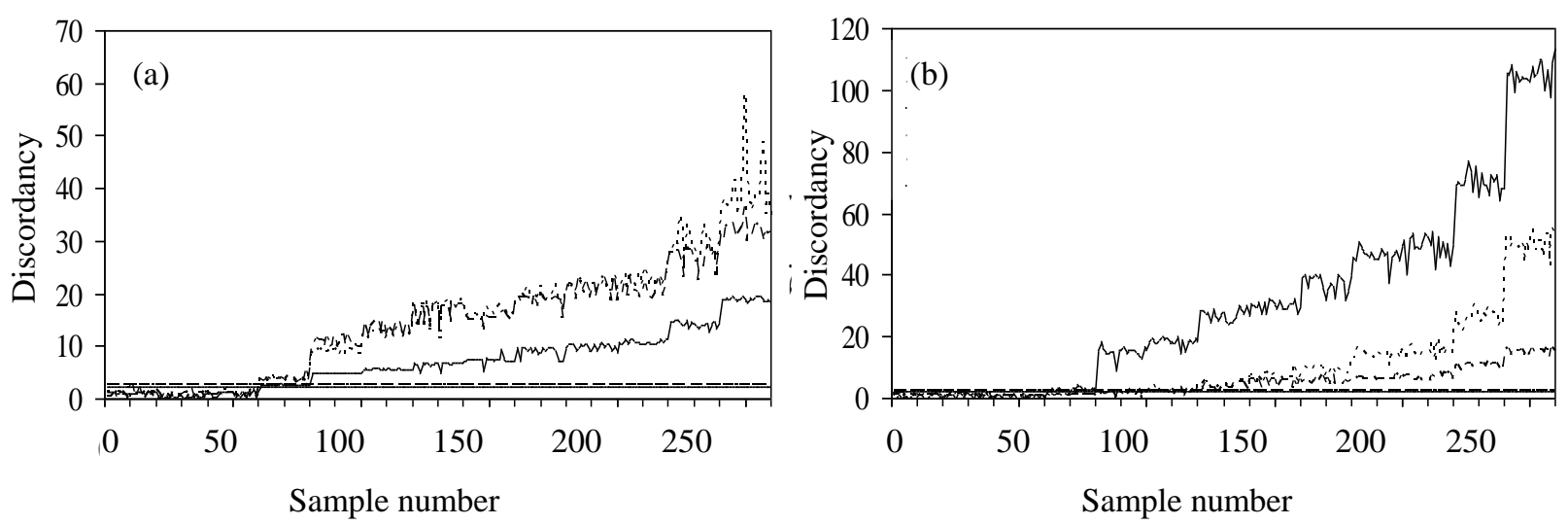

Figure 4 - Discordancy test for the features damage index root mean square, peak-to-peak, and K-factor from the wavelet coefficient vector calculated for (a) $\mathrm{A}_{0}$ mode at $225 \mathrm{kHz}$, (b) $\mathrm{S}_{0}$ mode at $225 \mathrm{kHz}$.

line). Peat-to-peak (dashed line). K-factor (dotted line).

\section{Nonlinear approach}

Sinusoidal acoustic waves at a chosen frequency and at five different amplitudes were applied (one amplitude at a time) to one of the PZT-patches (henceforth called transmitter) and the response was measured at another PZT-patch (henceforth called sensor). In Fig. 5, PZT-A serves as the transmitter and PZT-B as the sensor for the Western tension flange. A similar set of transducers were used for the Eastern tension flange. The frequency of excitation was chosen to be one of the resonant frequencies of the transducer-structure system. This ensured high amplitude of the travelling acoustic waves which would aid in the process of crack opening and closing and hence in the production of second harmonic due to crack. For each amplitude of excitation, the magnitude of the second harmonic of the exciting frequency was extracted from the Fast Fourier Transform (FFT) of the time-signal measured at the sensor. The measurements were performed after every few 
thousands cycles of the midspan loading (after $0,5 \mathrm{~K}, 10 \mathrm{~K}, 12 \mathrm{~K}, 14 \mathrm{~K}, 18 \mathrm{~K}, 22 \mathrm{~K}$ and $24 \mathrm{~K}$ cycles to be precise) when the loading was paused with a static load of $22 \mathrm{kN}$ sitting on the beam.

The resonant frequency of the transducer-structure system was measured once at the onset of loading and once after $12 \mathrm{~K}$ cycles when the crack already existed. Fig. 9 shows the amplitude spectrum of the transducer-structure system when a Gaussian white noise input was given to the transmitter. The forwarding signals were measured twenty times and averaged in the frequency domain to diminish noise effects. It can be observed from Fig. 9 that the resonant frequency of the cracked system did not vary significantly from the resonant frequency of the undamaged system. The driving frequency for all subsequent experiments was therefore chosen to be $350.5 \mathrm{kHz}$ for the set of transducers on the Western flange and $356 \mathrm{kHz}$ for those on the Eastern flange. The amplitude spectrum was not measured again due to time constraints.
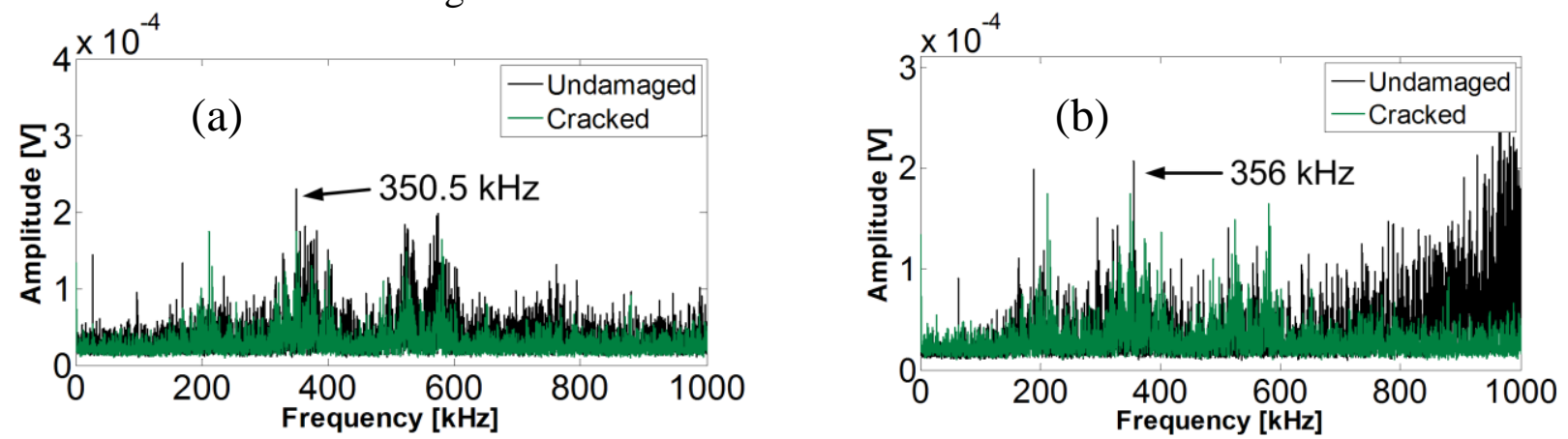

Figure 5 - Amplitude spectrum of the transducer-structure system when a Gaussian white noise input was given to the transmitter (a) Western tension flange (b) Eastern tension flange

Once the resonant frequency of the system was identified, a sinusoidal signal with a \pm 1 peak-topeak voltage and driving frequency equal to the resonant frequency of the system was generated using the AWG and applied to the transmitter. FFT of the response measured at the sensor was taken, and the absolute value of the FFT at the second-harmonic of the driving frequency was noted. The forwarding signals were again measured twenty times and averaged in the frequency domain. The above procedure was then repeated with the peak-to-peak excitation voltage varying from $\pm 4 \mathrm{~V}$ to $\pm 40 \mathrm{~V}$ with an incremental step of $\pm 9 \mathrm{~V}$.
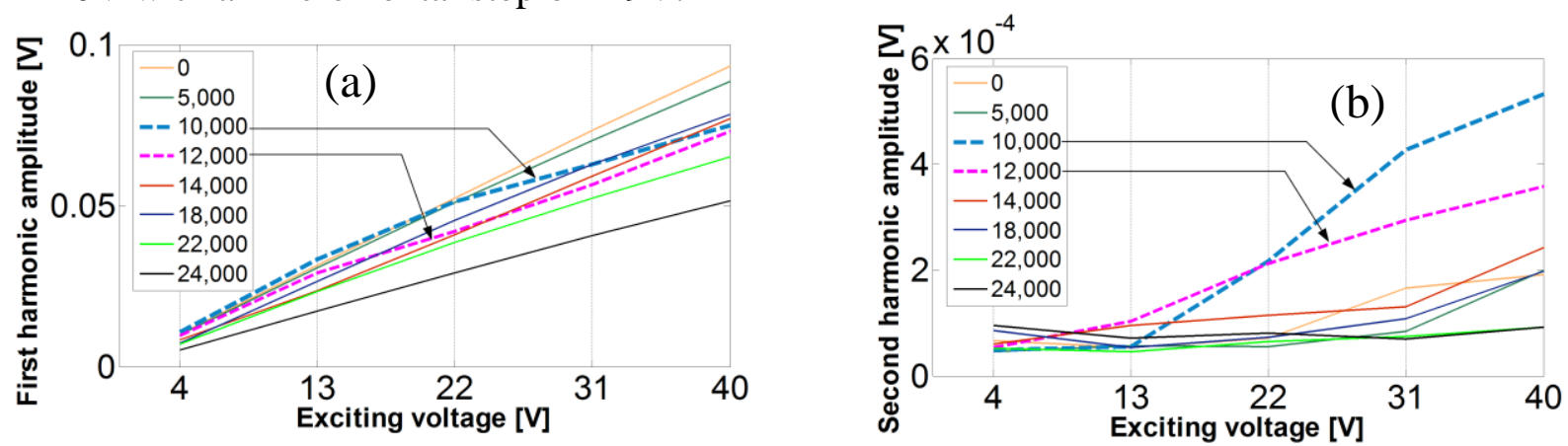

Figure 6 - Variation of (a) first harmonic $(350.5 \mathrm{kHz})$ and (b) second harmonic $(701 \mathrm{kHz})$ amplitudes in the signal measured at the sensor with increasing excitation voltage. Results are shown after given number of cycles of mid-span loading; Crack initiated around 8790 cycles. (Results from Western tension flange)

Fig. 6 shows the results from the Western tension flange. Fig. 6a suggests an increased acoustic nonlinearity at approximately ten thousand cycles when the first harmonic amplitude varies nonlinearly with the excitation voltage. The presence of the second harmonic is also significant around ten thousand cycles (Fig. 6b). These observations combined with observations from the crack gages (Fig. 3) imply that the PZT-based active sensing system could identify the damage near its inception. The nonlinearity effects became prominent at the inception of the crack but its manifestation decreased steadily with increasing length of the crack and became indiscernible after fourteen thousand cycles.

Similar results were observed for the Eastern flange (Fig. 7). However, in this case the nonlinear effects became prominent at around twenty thousand cycles whereas the crack initiated after about 
ten thousand cycles. In conclusion it may be said that cracks in the tested specimen were successfully identified at some point of their propagation using the principles of nonlinear acoustics. However, it is not clear at what stage of crack propagation the harmonics would be most visible.
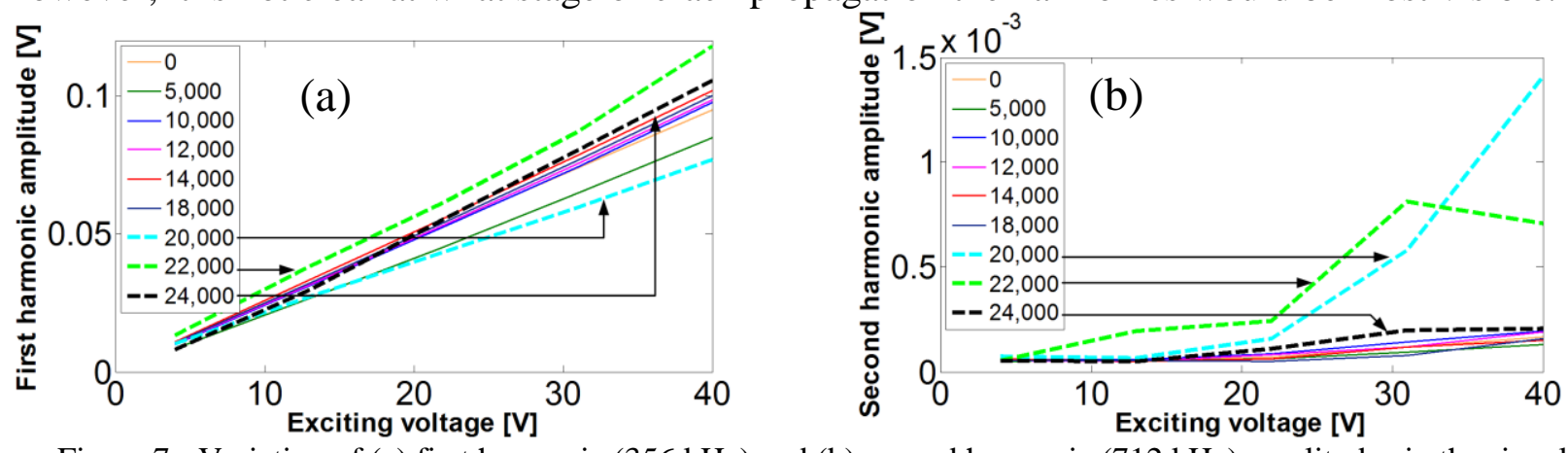

Figure 7 - Variation of (a) first harmonic $(356 \mathrm{kHz})$ and (b) second harmonic $(712 \mathrm{kHz})$ amplitudes in the signal measured at the sensor with increasing excitation voltage. Results are shown after given number of cycles of midspanloading; Crack initiated around 9500 cycles. (Results from Eastern tension flange)

\section{Conclusions}

This paper presents an automated crack detection technique for metallic waveguides using agile PZT transducers. Specifically, the study focused on the detection of the onset and propagation of fatigue crack induced on a steel structural beam. The technique is based on the propagation of linear and nonlinear ultrasonic guided waves. In the linear approach time waveforms were processed using discrete wavelet transform and univariate outlier analysis to detect anomalous conditions of the structure and in particular the presence of fatigue cracks. In the nonlinear approach preeminent harmonics in the response signal from cracked specimens were observed as the input power of the driving PZT-wafer increased. The harmonic amplitudes also showed nonlinear variation with the increasing excitation voltage in cracked specimens. The proposed technique identifies the presence of cracks by looking at two features: harmonics and their nonlinear relationship to the input amplitude. The structural health monitoring paradigm presented in this paper is applicable to many structural components having waveguide geometry (e.g. plates, rods, pipes) which lend themselves to guided ultrasonic wave propagation. Depending on the specific application, the ultrasonic configuration (whether pulse-echo or pitch-catch), the discrete wavelet transform decomposition levels, as well as the features considered in the computation of the damage index may change.

\section{Acknowledgements}

The support of the University of Pittsburgh to Mr. Cammarata is acknowledged. The third and fourth authors acknowledge the support of the Korea Science and Engineering Foundation (M20703000015-07N0300-01510) and Korea Research Foundation (D00462).

\section{References}

[1] T.M. Roberts and M. Talebzadehb: Acoustic J. Constr. Steel Res. 59(6) (2003), p. 695

[2] A. Sophian, G.Y. Tian, D. Taylor and J. Rudlin: Insight 43(5) (2001), p. 302

[3] T.G. Chondros, A.D. Dimarogonas and J. Yao: J.Sound Vib. 215(1) (1998), p. 17

[4] G. Park, H. Sohn, C.R. Farrar and D. J. Inman: Shock Vib. Dig. 35(6) (2003), p. 451

[5] J. W. Staszewski Adv. Smart Techn. Struct. Engr. (2003), p. 117.

[6] P. Rizzo and F. Lanza di Scalea Progress in Smart Materials and Structures Research, (NOVA publ. 2007), p. 227

[7] V. Giurgiutiu: J. Intell. Mat. Syst. Struct. 16(4) (2005), p. 291.

[8] D. Cook and Y. Berthelot: NDT\&E Intl. 34 (2001), p. 483

[9] W. Leong, W. Staszewski, B. Lee and F. Scarpa: Smart Mat. Struct. 14 (2005), p. 1387

[10] W. Staszewski, B. Lee and R. Traynor: Meas. Sci. Technol. 18 (2007), p. 727

[11] V. Barnett and T. Lewis Outliers in statistical data (New York: John Wiley \& Sons, 1984)

[12] I. Solodov, W. Yu., N. Krohn and G. Busse: Ultrasonics (2002), p. 621.

[13] O. Buck, W. Morris, and J.M. Richardson: Appl. Phys. Lett. 33(5) (1978), p. 371.

[14] Pecorari, C. (2003) : J. Acoust. Soc. Am. 113(6), 3065-3072. 